\title{
Condiciones políticas existentes para la fijación de tarifas de agua potable. Análisis desde la paradoja de CONDORCET
}

\author{
URL: http://revistas.uta.edu.ec/erevista/index.php/bcoyu/article/view/719 DOI: http://dx.doi.org/10.31164/bcoyu.22.2019.719

\begin{abstract}
Marco Piedra- Aguilera'; José Vera -Reino²; Ana Albornoz ${ }^{3}$; Camila Palacios ${ }^{4}$
\end{abstract} \\ Fecha de recepción: 18 de febrero de 2019 \\ Fecha de aceptación: 19 de agosto de 2019
}

\section{Resumen}

El artículo estudia la Paradoja de Condorcet aplicada al funcionamiento del Cabildo Cantonal de Cuenca - Ecuador. La votación realizada al interior de un espacio político obedece a la regla de mayorías aún cuando su decisión involucre el normal desenvolvimiento organizacional. Los hallazgos determinan que por varios años las tarifas de agua potable no fueron modificadas pese a que ETAPA-EP presentaba necesidades de dinero y veía comprometida su operación. Se reconoce la Paradoja de Condorcet cuando las preferencias de los votantes son cíclicas aún a pesar de que la preferencia individual sea contraria a la grupal. El estudio presenta análisis de tipo histórico - cualitativo.

Palabras clave: Empresa de agua, gobierno local, decisiones sociales, economía, política, fijación de tarifas.

\section{Abstract}

The article studies the Paradox of Condorcet applied to the operation of the Government of Cuenca - Ecuador. Voting carried out within a political space obeys the rule of majorities even when its decision involves normal organizational development. The findings determine that drinking water rates were not modified for several years despite the fact that ETAPA-EP presented needs for money and its operation was compromised. The Condorcet Paradox is recognized when the preferences of the voters are cyclical even though the individual preference is contrary to the group preference. The study presents historical-qualitative analysis.

Keywords: Water Company, local government, social decisions, economy, politics, rate setting.

\section{Introducción}

El presente trabajo pretende determinar la relación existente entre la composición del Cabildo de la ciudad de Cuenca y las decisiones adoptadas en la empresa de mayor importancia que le pertenecen al Gobierno Autónomo Descentralizado Cantonal, la cual, es proveedora de agua potable para la ciudad; en este contexto se realiza el análisis bajo el paraguas denominado la paradoja de Condorcet, y comprobar que la regla de las mayorías es válida, a pesar de que pueda llegarse a comprometer el normal desarrollo y la labor futura de esta empresa pública ETAPA-EP.

Se parte de datos proporcionados por dos empresas del sector público, la primera ETAPA-EP de la cual, se consiguieron los paquetes tarifarios aprobados y vigentes para los años de estudio; en segundo lugar, se mantuvo acceso a los registros históricos del Consejo Nacional Electoral (CNE, por sus siglas en español) desde el año 1998 hasta el 2014, para poder obtener los datos de la conformación de los cabildos en los años de estudio. La información tuvo un prolongado periodo de espera y, para el caso del CNE fue entregada incompleta, pues la entidad no contaba con los registros de las dignidades electas en ciertos periodos. El inconveniente fue solventado acudiendo a los registros históricos de las sesiones de Consejo Cantonal en los archivos de la Alcaldía de Cuenca. Es menester destacar que la empresa ETAPA-EP basa su importancia para Cuenca en virtud de estar al servicio desde hace más de 50 años, y ser un referente a nivel local como a nivel nacional en el Ecuador. Su red de distribución de agua potable alcanza una cobertura del 96\% en el área urbana y 88\% en el área Rural (epata.net.ec, 2019).

El apartado II muestra la revisión de literatura, misma que comienza con las definiciones básicas de lo que comprende el espectro de izquierda y derecha, y de cómo la paradoja de Condorcet se ha ido desarrollando a lo largo del tiempo. El apartado III presenta la metodología seguida en la investigación. El apartado IV hace una revisión de los principales resultados obtenidos, y culminamos con el apartado $\vee$ que muestra la conclusión a la que se llegó con el presente estudio.

\section{Revisión de Literatura}

Para un mejor entendimiento, es necesario la inclusión de ciertos términos partiendo del capitalismo; el cual, es un sistema social que parte de la obtención de beneficios de la propiedad privada y prevalece al capital sobre el trabajo y es la base de la generación de riqueza. La propiedad privada y el libre mercado son pilares fundamentales. (Smith, A. 1974), y luego incluimos a la denominada democracia participativa, cuya forma de gobierno expone que las funciones políticas son ejercidas por el pueblo (Ross, 1952), contrastando esta posición con la denominada democracia representativa que giran en torno a la transmisión representativa del poder elegidos a través del sufragio. (Sartori, 1998).

Adicionalmente, vale la pena definir lo que se denomina ideología política de izquierda y derecha, siendo los primeros los que dan mayor importancia a la condición igualitaria de los hombres o a las formas de atenuar y reducir los factores de desigualdad; y la ideología de derecha quienes están convencidos de que las desigualdades son un dato ineliminable. (Bobbio, 1996).

Brevemente abordemos a lo que se define como neoliberalismo, al cual se le clasifica como una teoría de prácticas político-económicas, y afirma que el bienestar del ser humano consiste en no restringir el libre desarrollo de las capacidades y el de las libertades empresariales, defendiendo la propiedad privada, y la libertad de comercio y de mercado (Harvey, 2005), al cual se le contrasta por tradición con el socialismo que se compone como un sistema de organización social y económica basado en la propiedad y administración colectiva o estatal de los medios (Diccionario de la Real Academia Española, 2014) cuyos representantes suelen proclamarse como partidos programáticos, pues ofrecen canales institucionales para la articulación del disenso social con respecto a la ortodoxia de mercado. (Roberts, 2013).

Es de común consenso el saber que los países sudamericanos han tenido complejos escenarios sociales, cuyas características predominantes han sido la explotación, el abandono, la dependencia y el subdesarrollo (Hamburger Fernández, 2014) condiciones que pese a haber aplicado el modelo de Industrialización por Sustitución de Importaciones (ISI) (Roberts, 2013) no dio el resultado esperado pues fue reemplazado en 1980 por un modelo neoliberal tras la crisis de la deuda externa generalizada en Latinoamérica que afectó a varias países de la región, fortaleciendo la ideología de izquierda en casi toda la región (Roberts, 2013), ideología que ofrece una combinación del socialismo con la democracia participativa y directa (Hamburger Fernández, 2014).

En este contexto de análisis, es importante también referirse a la evolución de sector público, el cual, ha desempeñado un papel fundamental en la concepción política económica de los estados modernos. Hegel (1821), considera al Estado no solo como un poder sino como un bien, siendo este el punto de partida para la necesidad de un sector publico planificado. En Ecuador, la planificación estatal comenzó en 1954 con el expresidente José María Velasco Ibarra bajo Decreto Ley de Emergencia (Jara Alba \& Umpierrez de Reguero, 2014) con la que se instaura la corriente de pensamiento del buen vivir que ha marcado los cambios en el modo de planificación actual.

En el caso particular de la ciudad de Cuenca, la empresa ETAPA - EP inicia sus actividades en octubre de 1945 cuando el Concejo Municipal de la época firmo un acuerdo con la compañía LM Ericsson (ETAPA EP, 2017), siendo en el año de 1948 cuando la empresa asume la responsabilidad en la dotación de agua potable para la ciudad (ETAPA EP, 2017), empresa que goza de patrimonio propio, dotadas de autonomía presupuestaria, financiera, económica, administrativa y de gestión ( Ediciones Legales, 2017), cuyo pliego tarifario último fue aprobado el 30 de noviembre del 2016 según consta en actas por el Concejo Cantonal de Cuenca y demás representantes (ETAPA EP, 2017).

Dentro de todo este espectro, se requiere aplicar un análisis que permita aplicar la regla de Condorcet en decisiones en donde se incluyen votaciones de grupos de personas que se obedecen a sectores políticos específicos (Blandón Restrepo, 2016), teniendo presente que esta decisión viene tomada por un grupo de personas y no de manera unimodal, (Downs, 1957) pues bajo estas condiciones el resultado del teorema de la mediana es el mismo que del ganador de Condorcet (Black, 1948), siendo la característica especifica de este método la de trabajar bajo un esquema ordinal (Mello, 2015). La pertinencia de la aplicación de Condorcet radica en el hecho de que esta herramienta permite validar la posición de las mayorías aun en detrimento de lo que pueda suceder en los entornos con las necesidades de la sociedad.

Se reconoce a la paradoja de Condorcet cuando las preferencias de los votantes son cíclicas aun a pesar de que la preferencia individual puede llegar a ser contraria a lo que se decide en grupo (Guerra-Pujol, 2016), siendo el caso analizado 
en el presente documento al momento de decidir sobre los servicios ofertados a la ciudad sobre la preferencia de un grupo de votantes y sobre las condiciones particulares financieras internas de la empresa proveedora del servicio (Guillermo, 30).

Cabe anotar que el método presenta ciertas debilidades, que pueden inclusive comprometer el normal desenvolvimiento de los temas en discusión predominando la irracionalidad (Goldoni, 2015), como en el presente caso la posición financiera de la empresa ETAPA EP (Castillo, 2014) evidenciando que las decisiones adoptadas bajo el enfoque de Condorcet resultan incoherentes con respecto a las que se adoptarían bajo un esquema racional (Piffano, 2009).

Por último, se define la manera en la que las leyes electorales declaran ganadores en los años de estudio (1.995 al 2017), teniendo que entre los años 1986 y el año 1998 primó según el artículo 85 de la ley electoral el cálculo de sistema de cociente y residuo electorales (Tribunal Supremo Electoral). La ley reformatoria de la Ley de Elecciones del año 1998 indica que para las elecciones pluripersonales se abandonó el sistema de votar por listas cerradas, y se las abrió para que el elector pueda escoger sus candidatos de una lista o entre listas". (Tribunal Supremo Electoral)

La Ley Orgánica de Elecciones del año 2.000 indica que se aplicará la fórmula D'Hont (Consejo Nacional Electoral, 2017). La Ley Orgánica de Elecciones de 2000 Reforma 2006 expone que se aplicará un método de divisores continuos. (Consejo Nacional Electoral, 2017).

El esquema vigente de adjudicación está declarado en el Código de la Democracia instaurado en el 2009, mismo que establece que para la adjudicación de listas se procederá de acuerdo con los cocientes mayores mediante la aplicación de la fórmula de divisores continuos y en cada lista, de acuerdo con quien haya obtenido las mayores preferencias. (Consejo Nacional Electoral, 2017).

\section{Metodología}

El trabajo emplea un estudio de tipo aplicativo / histórico documental de carácter cualitativo debido a su composición y tipo de información con la cual se va a trabajar, pues pretende establecer la condición existente en la ciudad de Cuenca en los últimos diecinueve (19) años en relación a la composición de su cabildo respecto a su espectro político y las decisiones que éste ha adoptado en cuanto al funcionamiento de una de sus empresas municipales, con el producto específico denominado agua potable en función de la fijación de sus tarifas residenciales, en el rango de consumo entre 0 a $20 \mathrm{~m}^{3}$ de líquido.

En primera instancia se recurrió a una revisión documental histórica por un lapso de 19 años y que tiene relación con la votación y nominación de los Concejales de la Ciudad de Cuenca, la cual tenía como finalidad el ubicar al cabildo en una posición ideológica dentro del espectro político denominado como de izquierda o de derecha; para lo cual, se solicitó un reporte escrito y avalado por el ente electoral que rige para la provincia del Azuay (Delegación Provincial Electoral del Azuay) quienes entregaron sus datos y fueron trabajados acorde al criterio descrito en primera instancia por composición de periodo y luego en función de línea de tiempo de los últimos 20 años.

El segundo componente implicó el acercamiento con los directivos de la empresa pública proveedora del servicio de agua potable ETAPA-EP a quienes se solicitó la entrega del pliego tarifario residencial que estuvo vigente desde el año 1995 hasta el año 2016, datos que fueron analizados y ordenados bajo el criterio de selección del pliego tarifario perteneciente a la categoría residencial, identificando las fechas específicas en las que tuvieron variaciones y estableciendo los periodos de tiempo que estos importes permanecieron sin alteraciones.

Con los datos obtenidos se procedió a aplicar un análisis estadístico el cual permitió determinar la correlación existente entre la composición del cabildo cantonal de Cuenca y la decisión de incrementar o no el pliego tarifario de agua potable de la categoría residencial.

A la información obtenida, se le analizó bajo el enfoque teórico de la paradoja de Condorcet (Caraballo, 2015) pretendiendo determinar el comportamiento cíclico del cabildo y sus decisiones, estableciendo preferencias de decisión de una empresa pública, el sostenimiento financiero de su producto agua potable en la categoría residencial, aplicando un gráfico donde aparece la relación entre dominante y dominada (Mello, 2015).

\section{Resultados}

El presente estudio se desarrolla bajo el enfoque de la paradoja de Condorcet por ser el más desarrollado y adaptado al momento de aplicar un análisis de votaciones múltiples (Merrill, 1988); a lo cual en un inicio el estudio planteaba que la estructura de gobierno cantonal tenia preferencias dadas (Blandón Restrepo, 2016), situación que es evidenciada al momento de analizar los datos históricos por medio de modelos estadísticos que perfilan estos postulados, manteniendo los resultados obtenidos en su enfoque originalmente planteado, puesto que el
Consejo Cantonal de la ciudad ha sido conformado por representantes de diversos bandos de la palestra política de la ciudad.

Los análisis requieren en primera instancia trabajar con las líneas de espectro político, con la cual, se declaran los partidos por los cuales, fueron electos los concejales que formaron parte del Cabildo en el periodo analizado. Otro dato que se toma en cuenta es la composición del Cabildo en función de las dignidades electas en su partido político original, no se consideran futuras desafiliaciones cambios de línea política de los involucrados. Al saber, la composición de cabildo fue analizada para los periodos comprendidos entre 1998 y la proyección al 2019 , en los cuales se han conformado 7 cabildos diferentes.

Es evidente que la composición de cabildo en la ciudad ha sido de tendencia de izquierda durante los últimos 19 años, siendo esta la característica que permite vislumbrar la posición de espectro político; con el que, se ha vivido en la ciudad. Sabiendo que cada nivel de gobierno es representado por varios agentes, en los cuales las mayorías han primado en función de la preferencia de proyectos que incluyeron votaciones de cada cabildo, nos permite definir la posición que adoptaron en relación con la votación por proyectos (Blandón Restrepo, 2016) y no las de afección a la ciudadanía.

La Figura 1, muestra la composición del cabildo en función de las dignidades electas, la cual expone que en el periodo de análisis 2014 - 2019 la mayoría de autoridades pertenecían a partidos denominados como de centro e izquierda, e periodo 2009 - 2014, al igual que los periodos 2007 - 2009; 2005 - 2007 y 2002 al 2005 estaba constituido por una mayoría de miembros que pertenecían en ese momento a partidos denominados como de izquierda. En el periodo comprendido entre el 2000 y el 2002 y el 1998 al 2000 se podría indicar que las fuerzas estaban divididas entre ideologías de izquierda y de derecha.

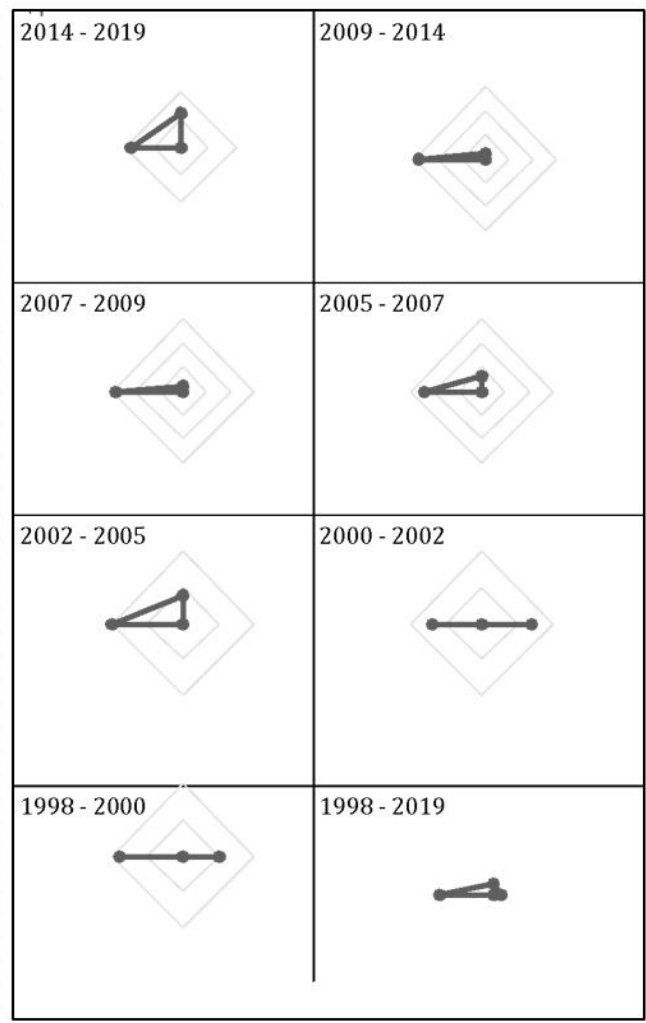

Fuente: (Consejo Nacional Electoral 2017) Figura 1. Composición del cabildo de Cuenca

En lo referente a agua potable se analiza la tarifa residencial en la categoría básica rango de consumo de $0-20 \mathrm{~m}^{3}$. La Tabla I, presenta el análisis de los años estudiados y las diferentes tarifas.

Cabe anotar que los datos presentados originalmente para los años 1998 y 1999 estaban expresados en Sucres (es moneda oficial del Ecuador), mismos que para efectos de comparación fueron transformados a dólares (moneda de circulación oficial a partir del año 2000) tomando como base su cotización en dichos periodos. 
Tabla 1. Años estudiados y tarifas básicas de consumo

\begin{tabular}{cccccc}
\hline AÑo & US/m3 & AÑo & US/m3 & AÑo & US/m3 \\
\hline 1998 & 0,02 & 2005 & 0,20 & 2012 & 0,20 \\
1999 & 0,03 & 2006 & 0,20 & 2013 & 0,20 \\
2000 & 0,07 & 2007 & 0,20 & 2014 & 0,20 \\
2001 & 0,09 & 2008 & 0,20 & 2015 & 0,20 \\
2002 & 0,18 & 2009 & 0,20 & 2015 & 0,40 \\
2003 & 0,20 & 2010 & 0,20 & 2016 & 0,21 \\
2004 & 0,20 & 2011 & 0,20 & 2017 & 0,415 \\
\hline
\end{tabular}

Fuente: (Etapa-EP, 2017)

La tendencia de incremento de las tarifas de agua potable permanece inmóvil durante 13 años, situación que comprometió el normal funcionamiento de ETAPA-EP (Castillo, 2014). La Figura 2, muestra la tendencia del incremento de las tarifas de agua potable.

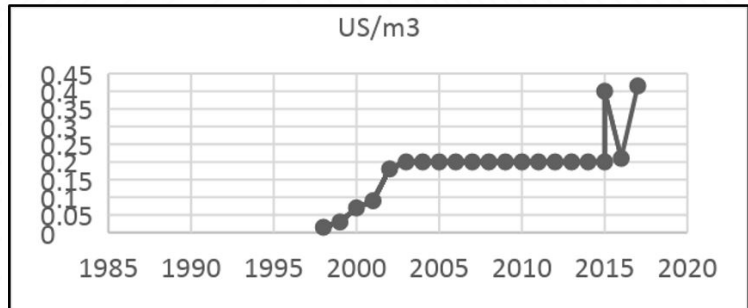

Figura 2. Incremento de tarifas de $\quad$ Fuente: (Etapa-EP, 2017)

Figura 2. Incremento de tarifas de agua potable residencial (1998 - 2017)
rende que para el año 2015, según la Sesión Ordinaria Incluso del análisis se desprende que para el año 2015, según la Sesión Ordinaria del Directorio de ETAPA del 30 de Abril de 2015 (M-2015-0468-GG) (Etapa-EP, 2017), se fija una tarifa superior a la mantenida en los últimos 13 años, misma de 2015 (M-0043-2016-SP) (Etapa-EP, 2017) retornando está a un valor muy cercano a su inicial (veinte centavos de dólar). Sin embargo, como el análisis es aplicado con relación a los periodos por los cuales los miembros del cabildo de la ciudad fueron electos, este valor quedaría como meramente informativo.

Para el año 2017, según la Sesión Ordinaria del Directorio de ETAPA del 30 de Noviembre de 2016 (M-0034-2017-SP) (Etapa-EP, 2017), cuando la situación financiera de la Empresa Publica, se convierte en critica, al cabildo no le queda otra opción más que tomar medidas de ajuste de tarifas, como bien lo indicaron sus funcionario al expresar que Etapa tiene un problema de liquidez, por eso "sinceró las tarifas" (Diario El Comercio, 2015)

Cuando se analiza el conjunto de los datos por medio de un análisis comparativo simple, se puede determinar que existe un criterio perfectamente relacionado entre el sector de espectro político de izquierda, y la no alza de las tarifas de agua potable. La Figura 3, muestra la relación del espectro político de los cabildos, y tarifas de agua.

\begin{tabular}{|c|c|c|}
\hline Periodo & Espectro & Impacto \\
\hline $1998-2000$ & & $367 \%$ \\
\hline $2000 \cdot 2002$ & $\leftrightarrow$ & $157 \%$ \\
\hline $2002-2005$ & $\infty$ & $11 \%$ \\
\hline $2005 \cdot 2007$ & $\rightarrow$ & $0 \%$ \\
\hline $2007 \cdot 2009$ & $\rightarrow$ & $0 \%$ \\
\hline $2009-2014$ & $\rightarrow$ & $0 \%$ \\
\hline $2014-2017$ & $\Delta$ & $108 \%$ \\
\hline
\end{tabular}

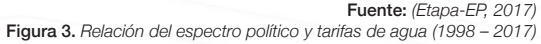

La relación existente entre las decisiones que se adoptan por parte de las autoridades responsables de la política de funcionamiento de las empresas públicas municipales y, en el presente caso con la de Etapa-EP y su principal producto, y el funcionamiento o rendimiento que estas puedan sostener, evidencia un comprometimiento inclusive del normal funcionamiento de las actividades para las cuales, deben las empresas sostenerse.

La información expuesta indica que el comportamiento del cabildo, debido a su composición de izquierda ha desarrollado sus actividades siguiendo los principios declaratorios ajustados a este tipo de ideología, supeditando e inclusive comprometiendo el desarrollo de las actividades de una empresa pública prestadora de un servicio básico, al no elevar los valores de la tarifa de agua.

Los resultados ratifican lo expresado por la regla de Condorcet en función de su enfoque de reglas de mayorías (composición de cabildo en el presente caso) (Blandón Restrepo, 2016) el mismo que ha permitido mantener inalterables los valores de las tarifas de agua potable para los ciudadanos de la ciudad de Cuenca en los últimos 19 años. Únicamente cuando el desenvolvimiento financiero de ETAPA - EP se vio comprometido, los involucrados decidieron votar a favor del incremento del valor del agua potable.

Sabiendo inclusive que el costo del agua cruda es cero (Roldán, 2016) en sus vertientes naturales, se concluye que el valor que es fijado por la empresa suministradora del servicio considera únicamente los gastos de potabilización, canalización, mantenimiento de redes y demás gastos y costos conexos con la prestación y distribución del servicio, no había llegado a ser cubierto, como bien los manifestó su gerente en una entrevista realizada (El Telégrafo, 2015).

\section{Conclusiones}

Recordando que la empresa ETAPA - EP es regida por un cuerpo administrativo independiente, se puede considerar como incoherente el haber permitido que la empresa acumule varios ceros a la cantidad de déficit presupuestario con tal de mantener el voto de las mayorías de sus autoridades regentes. Únicamente cuando las circunstancias fueron preocupantes, se llegó a adoptar la medida de incremento de la tarifa en el producto agua potable, comercializado por ETAPA-EP.

Se ratifica lo que indica Condorcet en su paradoja, pues la evidencia empírica demuestra el actuar de las personas cuando se obedecen a cuerpos regidos por mayorías, afirmando las ideas de una posible manipulación discrecional de la agenda política en lo referente a decisiones sociales en pro de los beneficios de quienes ostentan el poder en momentos determinados, aún a pesar de la existencia de gobiernos democráticamente electos.

El resultado de la investigación expone, que la regla de las mayorías está presente, aún a saber de qué existen cuestionamientos profundos de sus decisiones, ratificando lo anteriormente dicho cuando se indica que Condorcet obedece a la regla de las mayorías, como en el presente caso, de la conformación de los cabildos de la ciudad de Cuenca a lo largo de los últimos años.

Este estudio demuestra que se dejan de lado las preferencias unimodales y se convierten en actuares manipulables por las mayorías, respaldando de esta manera a las reglas sociales de la colectividad que eligieron a sus representantes del sector de espectro izquierdista.

Se observa además que el político de turno valora más el presente y acuerda adoptar políticas de mayorías en contextos de izquierda (como la voluntad de la mayoría de la ciudadanía cuencana) puesto que su futuro en relación a reelección es indefinido (Querubín, 2014), siendo estos espacios aquellos que corroboran la importancia que tiene el manejo de agenda en las decisiones colectivas; la elección por mayoría simple, es un método válido de elección pero puede estar asociado a este problema de "irracionalidad" del colectivo.

Se ha aplicado una herramienta con alto rigor académico para explicar aquellas decisiones de irracionalidad aparente, pero que en el fondo llevan cierta carga de objetividad, como lo es, el denominado costo político al cual se tienen que enfrentar aquellas personas cuando toman decisiones que van en perjuicio de una ciudad y sus habitantes.

En las líneas precedentes se ha podido aproximar el perfil del político cuanto éste actúa como un maximizador de algo, comprobando que existe una actitud que va al estilo de balance económico, si quita algo, va a buscar cómo ponerlo de nuevo. Al político le importa ganar la elección, pero más le importa que la política que el proponga sea la de su preferencia, aún al saber de la existencia del poder tras el poder, fase en la cual, la alternativa electa depende de cómo (y quién) se disponga el orden de votación. 


\section{Referencias}

Ediciones Legales. (15 de Septiembre de 2017). Obtenido de http://www. etapa.net.ec/Porta/s/O/TRANSPARENCIA/Literal-a2/LEY-ORGANICA-DE-EMPRESAS-PUBLICAS.pdf

Black, D. (1948). On the rationale of group decision-making. Journal of Political Economy, 23-34.

Blandón Restrepo, D. (2016). Implementación en la asignación de proyectos con las regalías en Colombia: una aproximación teórica. Desarrollo y Sociedad, 233-270.

Bobbio, N. (1996). Derecha e Izquierda. Madrid: Santillana.

Caraballo, A. F. (2015). Un paseo por la historia de la Teoría de Juegos. Boletín de Matemáticas, 77-95.

Castillo, L. (julio de 21 de 2014). Etapa limitará sus proyectos por un déficit. Diario El Comercio, pág. $2 b$.

Consejo Nacional Electoral. (2017). Página Oficial. Obtenido de http://cne. gob.ec/es/

Diario El Comercio. (04 de mayo de 2015). Las tarifas de agua potable suben en Cuenca. Obtenido de http://www.elcomercio.com/actualidad/tarifas-aguapotable-cuenca.html Collins.

Downs, A. (1957). An economic theory of democracy. Nueva York: Harper

El Telégrafo. (19 de noviembre de 2015). Reajuste en tarifas de agua potable de Cuenca se completa este mes. Diario El Telégrafo, pág. 6 A.

ETAPA EP. (18 de SEPTIEMBRE de 2017). ACTA 04 - 2015. Obtenido de http://www.etapa.net.ec/Portals/O/TRANSPARENCIA/Literal-s/ACTA\%20ABRIL. $P D F$

ETAPA EP. (15 de septiembre de 2017). Quienes somos. Obtenido de http:// www.etapa.net.ec/Quienes-somos/Informacion-General

ETAPA-EP. (2017). Oficio respuesta UDA / ETAPA-EP. Cuenca.

Goldoni, M. (2015). "Constitucionalismo político y el valor de la toma de. Derecho y Crítica Social, 67-107.

Guerra-Pujol, F. E. (9 de Octubre de 2016). Condorcet's Paradox in Puerto Rico. Obtenido de University of Central Florida; Pontifical Catholic University of Puerto Rico: $h$ ttp://dx.doi.org/10.2139/ssrn.2817959

Guillermo, A. (2014 de julio de 30). ETAPA: déficit supera los USD 38 millones. El Mercurio, pág. 4C.

Hamburger Fernández, Á. A. (30 de Abril de 2014). El socialismo del siglo xxi en américa latina: Características, desarrollos y desafíos. Recuperado el 10 de agosto de 2017, de Revista de Relaciones Internacionales, Estrategia y Seguridad-Universidad Militar Nueva Granada: https://revistas.unimilitar.edu.co/index. php/ries/article/view/54/1694

Hegel, G. (1821/1988). Principios de la filosofía del derecho o derecho natural y ciencia política. Barcelona, España: Edhasa.

Harvey, D. (2005). Breve historia del neoliberalismo. Madrid: Ediciones Akal.

Jara Alba, C., \& Umpierrez de Reguero, S. (29 de Diciembre de 2014). Evolución del sector público ecuatoriano desde 1998 a 2013. Revista Enfoques, XII, 131-148.

Mello, Á. S. (2015). Analysis of the multi-criteria decision-making Veto method. Ingeniare, 556-568.

Merrill, S. (1988). Making Multicandidate Elections More Democratic. Nueva Jersey: Princeton $\cup$ Press.

Roldán, D. F. (2016). Valoración económica de recursos hídricos para el suministro de agua potable. Tesis presentada para aspirar al grado de DOCTOR POR LA UNIVERSIDAD DE ALICANTE, 1-293.

Piffano, H. L. (2009). El Dilema de Condorcet - el Problema de la Votación por Mayoría Simple de Duncan Black - la Paradoja De Kenneth Arrow - y el Manejo de Agenda. Documento de Trabajo Nro. 76, 1-13.

Querubín, L. F. (29 de julio de 2014). Economía Política de la Política Económica. Obtenido de https://economia.uniandes.edu.co/files/jortegon@fundacionuniandes.edu.co/Leopoldo\%2OFergusson/Book_2013.pdf

Roberts, K. M. (11 de abril de 2013). Reforma de mercado, (des) alineamiento programático y estabilidad del sistema de partidos en América Latina. Salamanca, 163-191.

Ross, A. (1952). Why Democracy? Harvard University Press.

Sartori, G. (1998). En defensa de la representación política. Claves de razón práctica, 91.

Smith, A. (1794). La riqueza de las naciones. Oficina de Viuda e Hijos de Santander. Valladolid 1794.

Tribunal Supremo Electoral. (s.f.). Ecuador elecciones 1998. Obtenido de https://repositories. lib.utexas.edu/bitstream/handle/2152/17557/libro_4.pdf?sequence $=$. 